\title{
Scaffold-Free Fabrication of Osteoinductive Cellular Constructs Using Mouse Gingiva-Derived Induced Pluripotent Stem Cells
}

\author{
Hiroko Okawa, ${ }^{1,2}$ Hiroki Kayashima, ${ }^{2}$ Jun-Ichi Sasaki, ${ }^{3}$ Jiro Miura, ${ }^{4}$ \\ Yuya Kamano, ${ }^{1}$ Yukihiro Kosaka, ${ }^{1}$ Satoshi Imazato, ${ }^{3}$ Hirofumi Yatani, ${ }^{2}$ \\ Takuya Matsumoto, ${ }^{5}$ and Hiroshi Egusa ${ }^{1,2,6}$ \\ ${ }^{1}$ Division of Molecular and Regenerative Prosthodontics, Tohoku University Graduate School of Dentistry, Sendai, \\ Miyagi 980-8575, Japan \\ ${ }^{2}$ Department of Fixed Prosthodontics, Osaka University Graduate School of Dentistry, 1-8 Yamadaoka, Suita, Osaka 565-0871, Japan \\ ${ }^{3}$ Department of Biomaterials Science, Osaka University Graduate School of Dentistry, 1-8 Yamadaoka, Suita, Osaka 565-0871, Japan \\ ${ }^{4}$ Division for Interdisciplinary Dentistry, Osaka University Dental Hospital, 1-8 Yamadaoka, Suita, Osaka 565-0871, Japan \\ ${ }^{5}$ Department of Biomaterials, Graduate School of Medicine, Dentistry and Pharmaceutical Sciences, Okayama University, \\ 2-5-1 Shikada-cho, Kita-ku, Okayama 700-8525, Japan \\ ${ }^{6}$ Center for Advanced Stem Cell and Regenerative Research, Tohoku University Graduate School of Dentistry, Sendai, \\ Miyagi 980-8575, Japan
}

Correspondence should be addressed to Hiroshi Egusa; egu@dent.tohoku.ac.jp

Received 21 November 2015; Accepted 18 February 2016

Academic Editor: Boon C. Heng

Copyright (C) 2016 Hiroko Okawa et al. This is an open access article distributed under the Creative Commons Attribution License, which permits unrestricted use, distribution, and reproduction in any medium, provided the original work is properly cited.

\begin{abstract}
Three-dimensional (3D) cell constructs are expected to provide osteoinductive materials to develop cell-based therapies for bone regeneration. The proliferation and spontaneous aggregation capability of induced pluripotent stem cells (iPSCs) thus prompted us to fabricate a scaffold-free iPSC construct as a transplantation vehicle. Embryoid bodies of mouse gingival fibroblast-derived iPSCs (GF-iPSCs) were seeded in a cell chamber with a round-bottom well made of a thermoresponsive hydrogel. Collected ball-like cell constructs were cultured in osteogenic induction medium for 30 days with gentle shaking, resulting in significant upregulation of osteogenic marker genes. The constructs consisted of an inner region of unstructured cell mass and an outer osseous tissue region that was surrounded by osteoblast progenitor-like cells. The outer osseous tissue was robustly calcified with elemental calcium and phosphorous as well as hydroxyapatite. Subcutaneous transplantation of the GF-iPSC constructs into immunodeficient mice contributed to extensive ectopic bone formation surrounded by teratoma tissue. These results suggest that mouse GF-iPSCs could facilitate the fabrication of osteoinductive scaffold-free 3D cell constructs, in which the calcified regions and surrounding osteoblasts may function as scaffolds and drivers of osteoinduction, respectively. With incorporation of technologies to inhibit teratoma formation, this system could provide a promising strategy for bone regenerative therapies.
\end{abstract}

\section{Introduction}

Regeneration of large bone defects caused by trauma, tumor resection, or severe alveolar ridge resorption in dentistry is still a clinical challenge that awaits efficient tissue engineering protocols to achieve sufficient regeneration $[1,2]$. Recent approaches to fabricating tissue-engineered bone rely on the osteoinductive ability of transplanted cells seeded in exogenous scaffolds $[3,4]$. Although biomaterial scaffolds facilitate three-dimensional (3D) culture of osteogenic/progenitor cells ex vivo, they have also been associated with immunogenicity, unsatisfactory biological activity, enhanced inflammatory reactions, and uncontrollable cell-biomaterial interactions [5]. Therefore, a scaffold-free approach, in which biomimetic 3D bone tissues are fabricated as cell constructs, could be an attractive alternative for generation of tissueengineered transplants.

For bone tissue engineering, bone marrow-derived mesenchymal stem cells (MSCs) are currently the most popular cell source because of their easy collection and preferential 


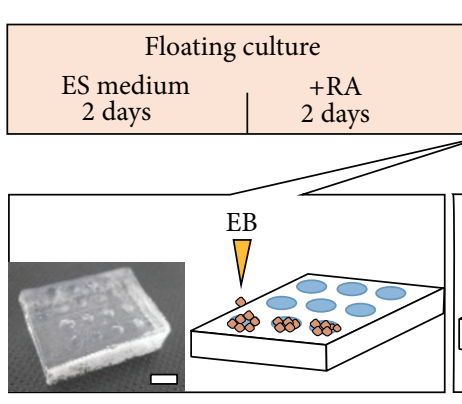

(b)

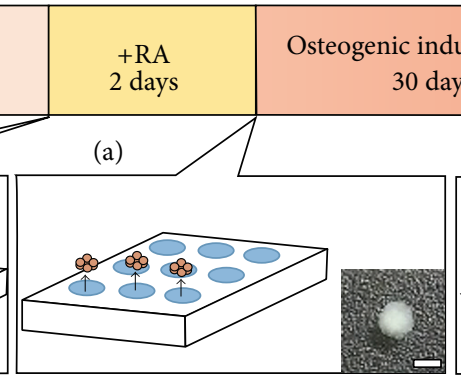

(c)

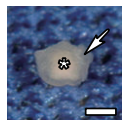

(e)

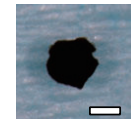

(f)

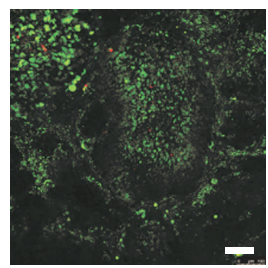

(g)

FIGURE 1: (a) Fabrication of osteogenic iPSC constructs. Mouse GF-iPSCs were cultured under floating conditions in ES medium for 4 days to form EBs. RA was added in the final 2 days. (b) EBs were seeded in wells of a pNIPAAm gel cell chamber (inset, scale bar: $5 \mathrm{~mm}$ ) and cultured for 2 days in ES medium with RA. (c) Ball-like cell constructs (inset, scale bar: $1 \mathrm{~mm}$ ) were collected by decreasing the temperature to expand the hydrogel chamber. (d) GF-iPSC constructs were cultured in osteogenic induction medium for 30 days with gentle shaking. (e) Osteogenically induced GF-iPSC construct on day 30 consisting of a white-colored core (asterisk) surrounded by a translucent layer (arrow). Scale bar: $1 \mathrm{~mm}$. (f) von Kossa staining of the osteogenically induced GF-iPSC construct. Scale bar: $1 \mathrm{~mm}$. (g) Live/Dead cell viability assay showed that most cells on the surface of osteogenically induced GF-iPSC constructs were alive with green fluorescence. A few cells were dead with red fluorescence. Scale bar: $100 \mu \mathrm{m}$.

differentiation to the osteogenic lineage [6,7]. Recently, MSCs have been applied to ex vivo fabrication of $3 \mathrm{D}$ osteogenic constructs in scaffold-based $[8,9]$ and scaffoldfree $[10,11]$ approaches. These osteogenic 3D constructs are expected to be effective osteoinductive materials, although the customization of the shape and size of the $3 \mathrm{D}$ cell constructs remains a challenge. In addition, laboratorygrown constructs, especially scaffold-free cell constructs, for bone regeneration often require a large amount of cells. In this regard, incidental cellular senescence and the limited proliferation capacity of MSCs may restrict their clinical application [12].

Induced pluripotent stem cells (iPSCs), which can be generated via genetic manipulation of somatic cells [13], possess pluripotency and unlimited proliferation capacity similar to that of embryonic stem (ES) cells. We previously reported that gingival fibroblasts (GFs) are a promising source of iPSCs in regenerative dentistry because they provide efficient generation of iPSCs [14] and can simultaneously be used as excellent autologous feeder cells [15]. Recent reports have demonstrated the osteogenic differentiation and bone formation ability of iPSCs [16]; however, no study to date has examined the potential use of iPSCs as scaffoldfree osteogenic 3D constructs. In suspension culture, iPSCs inherently form cell aggregates known as embryoid bodies (EBs). We previously reported that an osteogenic induction method for mouse GF-derived iPSCs (GF-iPSCs) in EBs was advantageous for osteogenesis, as the resulting iPSCs showed significantly higher calcium production capacity than MSCs during osteogenic differentiation [17]. We also established a method to obtain the desired size and morphology of 3D cell constructs using a temperature-responsive hydrogel [18].

In this study, we hypothesized that the high proliferation, aggregation, and osteogenesis capabilities of mouse GF-iPSCs would facilitate the fabrication of scaffold-free 3D osteogenic constructs. The objectives of this study were to fabricate 3D osteogenic iPSC constructs using EBs without scaffolds and to investigate their osteoinductive capability in an ectopic bone formation model.

\section{Materials and Methods}

2.1. Fabrication of $3 D$ GF-iPSC Constructs. The thermoresponsive poly-N-isopropylacrylamide (pNIPAAm) gel mold used as a cell chamber (diameter of $1.5 \mathrm{~mm}$ for each well) was prepared as previously described $[10,18,24]$. Mouse GFiPSCs that had been previously generated using retroviral introduction of Oct3/4, Sox2, and Klf4 (without c-Myc) [14] were expanded in 6-well plates on SNLP76.7-4 feeder cells. EB culture of iPSCs was performed on low-attachment culture dishes for two days in ES medium (DMEM with 15\% FBS, $2 \mathrm{mM}$ L-glutamine, $1 \times 10^{-4} \mathrm{M}$ nonessential amino acids, 1 $\times 10^{-4} \mathrm{M} 2$-mercaptoethanol, $50 \mathrm{U}$ penicillin, and $50 \mu \mathrm{g} / \mathrm{mL}$ streptomycin), followed by additional floating culture for 2 days in ES medium supplemented with $1 \mu \mathrm{M}$ all-transretinoic acid (RA; Sigma, MO) [17] (Figure 1(a)). The suspended EBs were seeded in each well of the pNIPAAm gel mold $(20 \mathrm{EBs}$; $4 \times 10^{6}$ cells/well) in ES medium supplemented with $1 \mu \mathrm{M}$ RA (Figure 1(b)). After two days of culture, cell constructs with a ball-like morphology were collected by decreasing the temperature from $37^{\circ} \mathrm{C}$ to $25^{\circ} \mathrm{C}$ (Figure $1(\mathrm{c})$ ).

2.2. Osteogenic Induction of iPSC Constructs. For osteogenic induction, the GF-iPSC constructs were cultured in a $60 \mathrm{~mm}$ dish in osteogenic induction medium [17] consisting of $\alpha$-MEM supplemented with $15 \%$ FBS, $0.1 \mu \mathrm{M}$ dexamethasone, $10 \mathrm{mM} \beta$-glycerophosphate, $50 \mu \mathrm{M}$ ascorbate-2phosphate, $100 \mathrm{U} / \mathrm{mL}$ penicillin, $100 \mu \mathrm{g} / \mathrm{mL}$ streptomycin, and $250 \mathrm{ng} / \mathrm{mL}$ amphotericin $\mathrm{B}$ for 30 days. To prevent the GF-iPSC constructs from adhering to the culture dish, shaking culture $[10,24]$ was performed using a seesaw shaker at $0.5 \mathrm{~Hz}$ (Figure $1(\mathrm{~d})$ ). The culture medium was changed every 2 days. 
TABLE 1: Primers used for SYBR Green quantitative RT-PCR.

\begin{tabular}{|c|c|c|c|}
\hline Description (gene name) & Primers (Fw, forward; Rv, reverse) & Product size (bp) & Accession number (reference) \\
\hline \multirow{2}{*}{ Runx2 (Runx2) } & Fw: 5'-CGGGCTACCTGCCATCAC-3' & \multirow{2}{*}{78} & \multirow{2}{*}{ NM_001146038.2 (Speer et al. [19]) } \\
\hline & Rv: $5^{\prime}$-GGCCAGAGGCAGAAGTCAGA-3' & & \\
\hline \multirow{2}{*}{ osterix $(S p 7)$} & Fw: 5' -CTCGTCTGACTGCCTGCCTAG-3' & \multirow{2}{*}{84} & \multirow{2}{*}{ NM_130458.3 (Fowlkes et al. [20]) } \\
\hline & Rv: 5'-GCGTGGATGCCTGCCTTGTA-3' & & \\
\hline \multirow{2}{*}{ collagen 1a1 (Colla1) } & Fw: $5^{\prime}$-TGTCCCAACCCCCAAAGAC-3' & \multirow{2}{*}{92} & \multirow{2}{*}{ NM_007742.3 (Kaback et al. [21]) } \\
\hline & Rv: $5^{\prime}$-CCCTCGACTCCTACATCTTCTGA-3' & & \\
\hline \multirow{2}{*}{ osteocalcin (Bglap) } & Fw: 5'-CCGGGAGCAGTGTGAGCTTA-3' & \multirow{2}{*}{68} & \multirow{2}{*}{ NM_007541.3 (Jadlowiec et al. [22]) } \\
\hline & Rv: 5'-AGGCGGTCTTCAAGCCATACT-3' & & \\
\hline \multirow{2}{*}{ GAPDH (Gapdh) } & Fw: 5' -TGCACCACCAACTGCTTAG-3' & \multirow{2}{*}{177} & \multirow{2}{*}{ NM_001289726.1 (Gautier et al. [23]) } \\
\hline & Rv: $5^{\prime}$-GGATGCAGGGATGATGTTC-3' & & \\
\hline
\end{tabular}

2.3. Reverse Transcription Polymerase Chain Reaction (RTPCR) Analysis. Quantitative real-time RT-PCR analysis was performed as previously described [17]. Following total RNA isolation (RNeasy Mini Kit: Qiagen, Hilden, Germany) and DNase I treatment (Ambion, Austin, TX), cDNA was synthesized from $1 \mu \mathrm{g}$ of total RNA using SuperScript III reverse transcriptase (Life Technologies). The SYBR Green assay was performed using Thunderbird SYBR qPCR Mix (Toyobo, Osaka, Japan) on a StepOnePlus real-time PCR system (Applied Biosystems). The primer pairs used are shown in Table 1 . The thermal profile of the PCR was $95^{\circ} \mathrm{C}$ for $10 \mathrm{~min}$, followed by 40 cycles at $95^{\circ} \mathrm{C}$ for $15 \mathrm{~s}$ and $60^{\circ} \mathrm{C}$ for $1 \mathrm{~min}$. Target gene expression was quantitatively analyzed using the $\Delta \Delta \mathrm{Ct}$ method [25].

2.4. Histochemical Staining. After osteogenic induction, GFiPSC constructs were fixed with $10 \%$ formalin neutral buffer solution for 7 days. After decalcification in Plank-Rycho solution, the specimens were embedded in paraffin for standard hematoxylin and eosin (H\&E) staining and Alcian blue staining. For immunofluorescent staining, deparaffinized sections were incubated in $0.05 \%$ Triton-X and 5\% skim milk (BD, $\mathrm{NJ}$ ) in phosphate-buffered saline at room temperature for $60 \mathrm{~min}$. After washing, the sections were incubated with antihypoxia-induced factor-1 $\alpha$ (HIF- $1 \alpha$ ) monoclonal antibody (H1alpha 67: 1/25, Santa Cruz Biotechnology, CA), antitype II collagen monoclonal antibody (5B2.5: 1/100, Abcam, Cambridge, UK), anti-osteocalcin polyclonal antibody (FL95: 1/100, Santa Cruz Biotechnology), or control IgG [normal mouse IgG (sc-2025) or rabbit IgG (sc-2027): 1/100, Santa Cruz Biotechnology] at $4^{\circ} \mathrm{C}$ overnight and then incubated for $60 \mathrm{~min}$ at room temperature with Alexa Fluor 488-conjugated goat anti-mouse IgG (1/500, Molecular Probes, Thermo Fisher Scientific, MA) or Alexa Fluor 555-conjugated goat anti-rabbit IgG (1/500, Thermo Fisher Scientific), followed by Hoechst 33258 (1/500, Thermo Fisher Scientific) nuclear staining. Cell viability on the surface of GF-iPSC constructs was assessed using the Live/Dead viability/cytotoxicity kit (Thermo Fisher Scientific) as previously described [26].

2.5. Characterization of Minerals Precipitated in the Osteogenic GF-iPSC Constructs. Specimens were fixed with 2\% paraformaldehyde and 2.5\% glutaraldehyde. After dehydration in an ascending series of ethanol, specimens were embedded in epoxy resin for standard methylene blue and von Kossa's calcium staining. Energy-dispersive X-ray spectroscopy (EDX) and selected area election diffraction (SAED) analyses were performed to characterize the minerals on the surface of the GF-iPSC constructs and to determine the presence of hydroxyapatite, respectively, as previously described [17].

2.6. Ectopic Bone Formation Assay. After osteogenic induction, 10 GF-iPSC constructs were mixed with $200 \mu \mathrm{L}$ of $40 \mathrm{mg} / \mathrm{mL}$ fibrinogen solution (Sigma), followed by addition of $200 \mu \mathrm{L}$ of $25 \mathrm{U} / \mathrm{mL}$ thrombin solution (Sigma) and incubation for 30 minutes at $37^{\circ} \mathrm{C}$ under $5 \% \mathrm{CO}_{2}$ to form a fibrin gel. The fibrin gel containing GF-iPSC constructs was subcutaneously transplanted into the dorsal skin of fiveweek-old immunodeficient mice (CB-17 SCID; Clea Japan, Tokyo, Japan). After 4 weeks, the transplants were extracted and fixed to prepare sections. Decalcified and nondecalcified sections were subjected to standard H\&E staining and von Kossa staining, respectively.

2.7. Statistical Analyses. A one-way analysis of variance (ANOVA) with Dunnett post hoc test was used for comparisons in the RT-PCR analysis. A significant difference was defined when $P<0.05$.

\section{Results and Discussion}

Prior to osteogenic induction, we cultured the EBs in the presence of RA $[17,27,28]$ to guide the mouse GF-iPSCs to initially differentiate into immature mesenchymal cells. We previously demonstrated that thermoresponsive pNIPAAm gels can be used to fabricate 3D cell constructs in which cell-cell and cell-matrix interactions are maintained [18]. When the RA-treated EBs were cultured in the roundbottom wells of the pNIPAAm gel chamber for two days, the EBs aggregated to form ball-like 3D cell constructs with the same diameter as the wells $(1.5 \mathrm{~mm})$ (Figure $1(\mathrm{c})$ : inset). During osteogenic induction, the size of the cell constructs gradually increased to approximately 1.7 -fold of 
the initial diameter (diameter of $2.60 \pm 0.37 \mathrm{~mm}$; average of 14 constructs) on day 30. On visual inspection, the osteogenically induced ball-like cell construct appeared to have a two-layer structure, consisting of a white-colored core surrounded by a translucent layer (Figure 1(e)). The cell construct had a black ball morphology on von Kossa staining (Figure 1(f)), suggesting that it was calcified. The calcified GFiPSC constructs were only obtained when the ball-like cell constructs were cultured in the osteogenic induction medium and not in the ES (growth) medium. In the EB medium, the ball-like cell constructs became soft and fragile, and they did not maintain their ball-like morphology, possibly because the GF-iPSCs differentiated into many different cell types. In the Live/Dead viability assay, most cells on the surface of the calcified GF-iPSC constructs showed intense green fluorescence (Figure $1(\mathrm{~g})$ ), indicating that they were viable.

We next used RT-PCR to analyze the expression of osteogenic marker genes (Runx2, osterix, collagen 1a1, and osteocalcin) in the viable cells of cell constructs during osteogenic induction. Expression of Runx2 (Figure 2(a)) and osterix (Figure 2(b)), which are key transcription factors for osteogenic initiation $[29,30]$, increased by more than 15 -fold on day 20 and day 10 , respectively. In parallel with the upregulation of these transcription factors, expression of collagen 1a1 (Figure 2(c)), a primary product of osteoblasts [31], and osteocalcin (Figure 2(d)), encoding the most abundant noncollagenous protein of bone matrix [32], was significantly increased by approximately 7-fold and 150-fold, respectively, at day 20 . These results suggest that the GF-iPSCs of the constructs were guided to differentiate robustly into osteoblastic cells under the osteogenic induction condition. It should be noted that increased expression of Runx2 occurred after upregulation of osterix. Although Runx2 is a key transcriptional factor for osteogenesis, osterix exerts its osteogenic function via Runx2independent mechanisms [33-35] and upregulation of osterix during osteogenesis may thus be independent of upregulation of Runx2. This mechanism may partly explain the unique expression pattern of Runx 2 and osterix during the osteogenic induction of the GF-iPSC constructs in the present study.

$\mathrm{H} \& \mathrm{E}$ staining of decalcified sections showed that the cell constructs basically consisted of two different structural regions (Figure 3(a)). The outer region was an osseous-like tissue with nucleated cells that were embedded in abundant bone-like extracellular matrix (ECM) (Figure 3(b)). Monolayer or multilayered cells were aligned on the outer and inner surfaces of the outer region (Figure 3(b)), as also confirmed by Alcian blue staining (Figure 3(c)). The inner region of the construct did not have a bone-like structure; rather, it contained an unstructured cell mass and some cells lacked nuclei (Figures 3(a) and 3(b)). In particular, the nucleus was missing in many cells in the center area, implying cell necrosis that possibly resulted from a low oxygen level [10]. In mouse MSC constructs fabricated using a mold of the same size as in the present study, we previously found that hypoxia and osteogenic induction guided the cells in the inner region to differentiate into hypertrophic chondrocytes through upregulation of the hypoxia marker HIF-1 $\alpha$ and the chondrogenic marker type II collagen [10]. In contrast, chondrogenic induction did not appear to occur in the
iPSC construct in the present site, based on the lack of Alcian blue staining (Figure 3(c)). In addition, expression of HIF- $1 \alpha$ was observed throughout the GF-iPSC construct, except in the center part (Figure 3(e)), whereas expression of type II collagen was mainly limited to the outer aligned cells and a few cells in the osseous-like region (Figure 3(f)). Although type II collagen is a cartilaginous ECM molecule, it is also expressed by skeletal stem/progenitor cells and their osteogenic progeny to regenerate bone [36]. The cells expressing type II collagen in the present study may thus have been osteogenic progeny that could have contributed to formation of osseous-like tissue inside the construct, thereby increasing the size of the construct by producing abundant osteogenic ECM. Indeed, the aligned cells and cells in the osseous-like region showed clear expression of osteocalcin (Figure 3(h)), which is secreted by osteoblasts as a bone matrix protein [37].

We next characterized the calcification of the GF-iPSC construct using nondecalcified specimens. von Kossa staining demonstrated positive staining in the innermost area of the cell construct (Figure 4(a)), indicating the presence of a calcified core. Expression of osteocalcin was observed throughout the inner region of the construct (Figure 3(h)), where expression of HIF-1 $\alpha$ was also observed (Figure 3(e)). The staining with the nonspecific IgGs was negative (Figures $3(\mathrm{~d})$ and $3(\mathrm{~g})$ ), confirming that the positive staining indeed showed expression of HIF-1 $\alpha$ (Figure 3(e)) and osteocalcin (Figure 3(h)) in the inner region of the GF-iPSC construct. MSCs subjected to hypoxia are more prone to differentiate into osteoblasts than those cultured in normoxic conditions [38], and the hypoxia-enhanced osteogenesis of MSCs is dependent on HIF-1 $\alpha$ [39]. HIF- $1 \alpha$ also mediates the stimulation of cartilage and vascular mineralization by osteocalcin [40]. Therefore, expression of HIF- $1 \alpha$ and osteocalcin induced by hypoxia and osteogenic induction may have been responsible for the robust mineralization of the immature mesenchymal cells in the internal region of the construct in the present study. During mineralization, the low oxygen level in the inner region likely caused most of the calcified cells to undergo necrotic cell death, resulting in a mineralized core in the construct with abundant bone ECMs including osteocalcin.

The inner areas of the construct were surrounded by a layer zone that was strongly positive on von Kossa staining (Figure 4(b)), suggesting the presence of a calcified bone matrix in the outer region of the construct that should be a part of the osseous-like tissue observed in H\&E staining. Osteoid-like tissues were also observed at the surface of the construct by methylene blue counterstaining. The distribution of elemental calcium and phosphorous was evaluated by EDX analysis in the outer region of the cell construct (Figure 4(c)), where high peaks of the EDX spectrum were confirmed, corresponding to elemental phosphorous and calcium (Figure 4(d)). We further evaluated hydroxyapatite formation in the calcified region by SAED analysis. TEM images of the outer region showed many electron-dense vesicles of needle-like mineral aggregates (Figure 4(e)), in which a clear diffraction ring pattern that represented the typical reflections of hydroxyapatite crystals [41] was demonstrated 


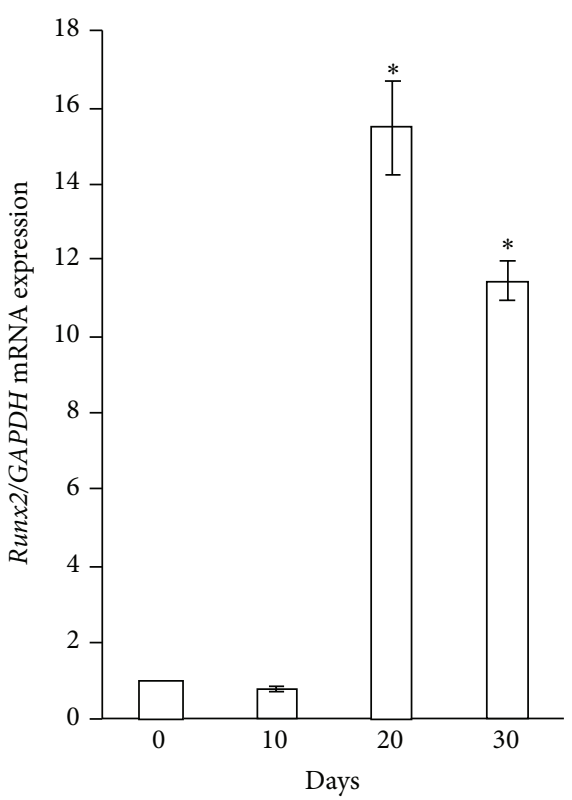

(a)

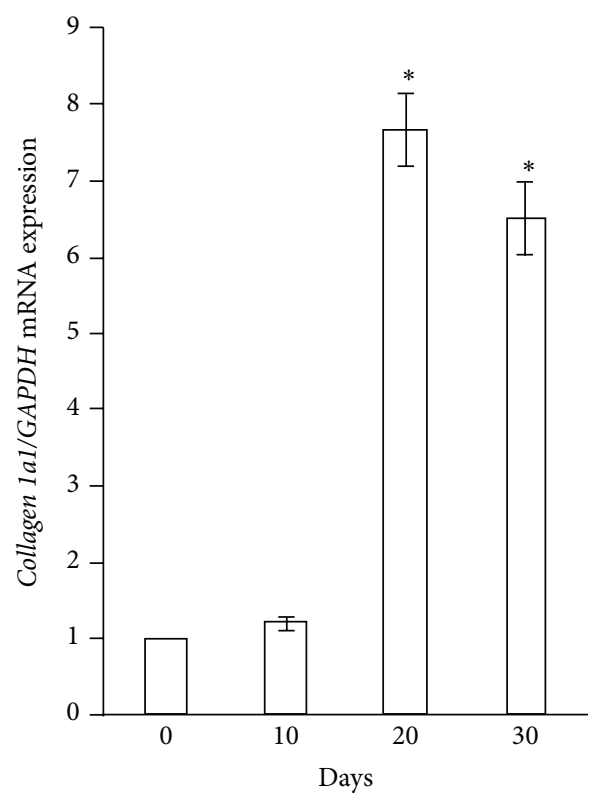

(c)

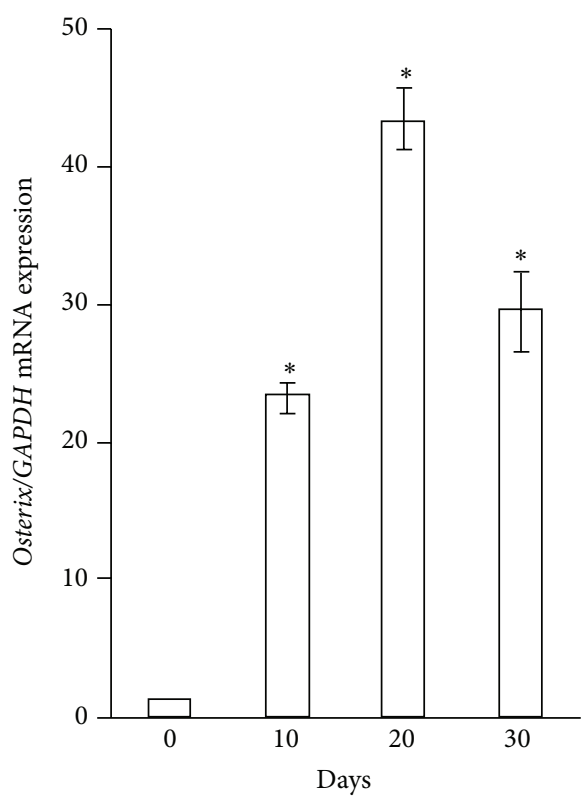

(b)

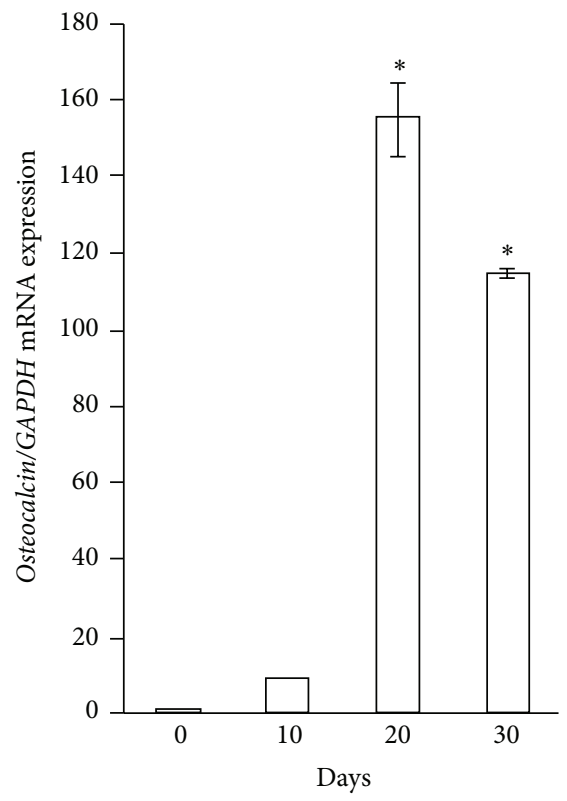

(d)

FiguRE 2: Expression of osteogenic marker genes in the GF-iPSC constructs under osteogenic induction. Expression of Runx2 (a), osterix (b), collagen 1a1 (c), and osteocalcin (d) on day 10,20, and 30 was determined by quantitative real-time RT-PCR. Gene expression of glyceraldehyde3-phosphate dehydrogenase (GAPDH) was used as an internal control. The data represent the mean values $\pm \mathrm{SD}(n=3)$. Significant differences $\left({ }^{*} P<0.01\right.$ : ANOVA with Dunnett's correction for multiple comparisons) were evaluated with respect to day 0 (before osteogenic induction) values.

(Figure 4(f)). These results suggest that we successfully fabricated ball-like 3D calcified cell constructs from mouse GF-iPSCs by a scaffold-free method, and these constructs consisted of a mineralized core and calcified osseous-like tissue surrounded by living osteogenic cells. Langenbach et al. [42] previously reported outgrowing cells from scaffold-free osteogenic microspheres that were fabricated using human umbilical cord blood-derived multipotent stem cells and thus inferred that the osteogenic microspheres could serve as a scaffold because of the accumulated collagen and its mineralization, whereas the outgrowing cells could be a source of osteogenic cells. Therefore, in our system, the aligned cells in the outermost layer of the GF-iPSC construct might serve as osteoinductive cells to form new bone in vivo.

To test this possibility, we investigated the osteoinductive capability of the GF-iPSC constructs in an ectopic bone 


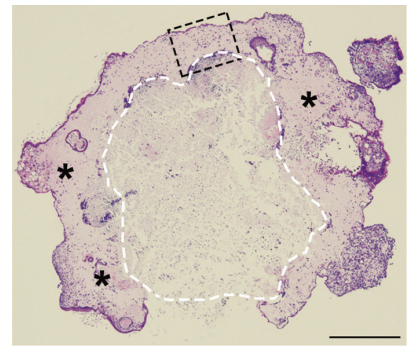

(a)

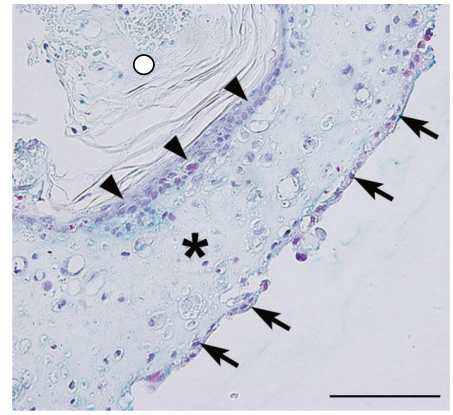

(c)

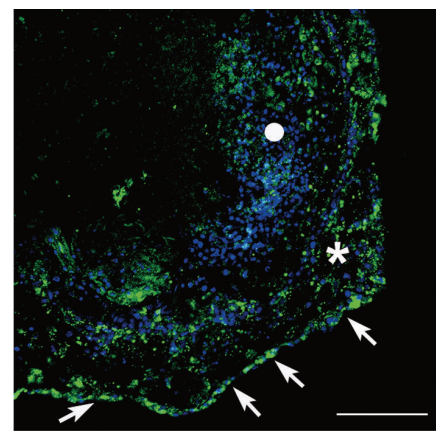

(e)

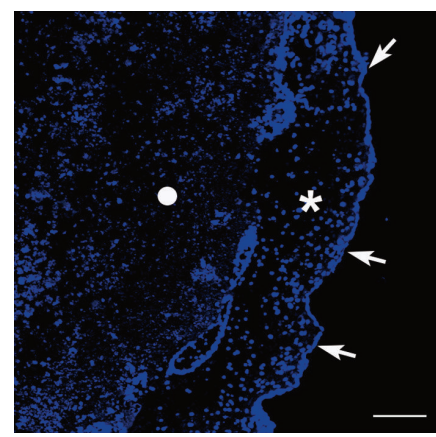

(g)

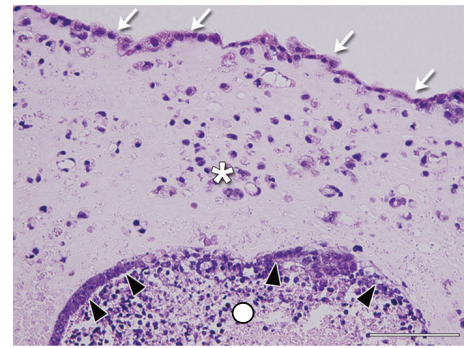

(b)

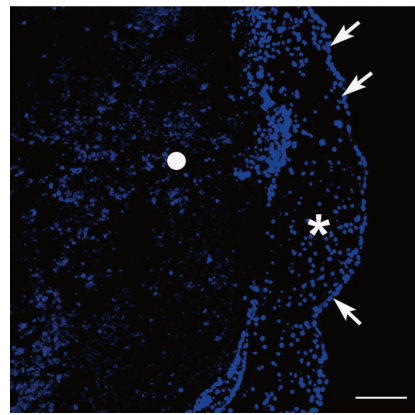

(d)

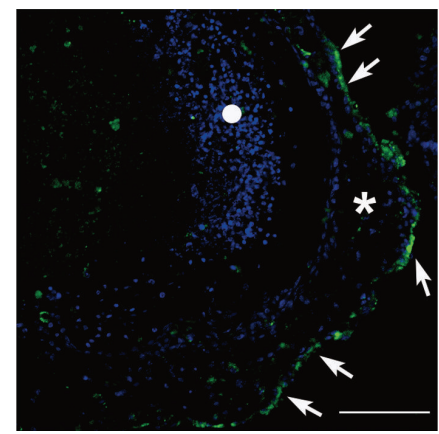

(f)

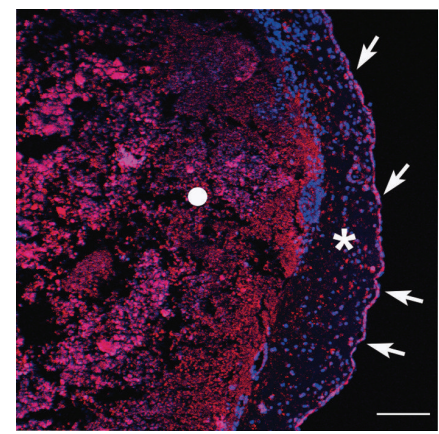

(h)

FIGURE 3: (a) H\&E staining of the osteogenically induced GF-iPSC construct, which consisted of an inner region of unstructured cell mass (inside the white dotted area) and outer osseous-like tissue region (asterisks). Scale bar: $0.5 \mathrm{~mm}$. (b) Magnification of the dotted square in panel (a). Aligned cells were present outside (arrows) and inside (arrow heads) the osseous-like region (asterisk). White circle indicates the inner region of the GF-iPSC construct. Scale bar: $100 \mu \mathrm{m}$. (c) Alcian blue staining also indicates the presence of aligned cells outside (arrows) and inside (arrow heads) the osseous-like region (asterisk). White circle indicates the inner region of the GF-iPSC construct. (d)-(h) Staining for HIF-1 $\alpha$ ((e) green fluorescence), type II collagen ((f) green fluorescence), osteocalcin ((h) red fluorescence), and nuclei ((d)-(h) blue fluorescence). Staining with nonspecific control IgGs as primary antibodies was used as a negative control (d and g). Aligned cells (arrows), osseous region (asterisks), and inner region (circles) of the cellular construct are indicated. Scale bars: $100 \mu \mathrm{m}$. 


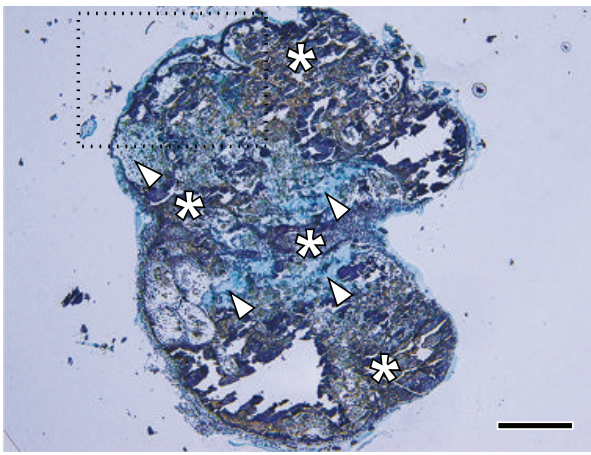

(a)

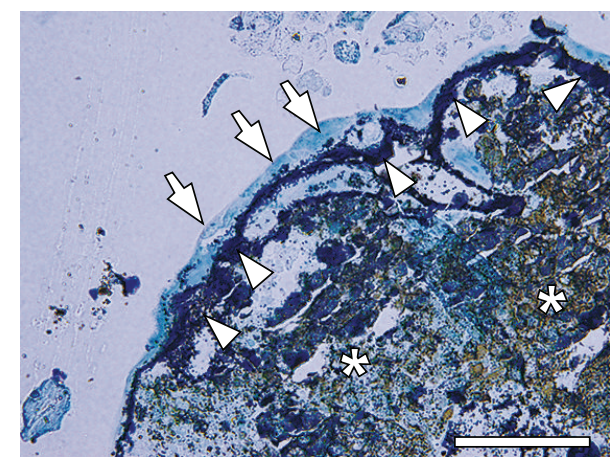

(b)

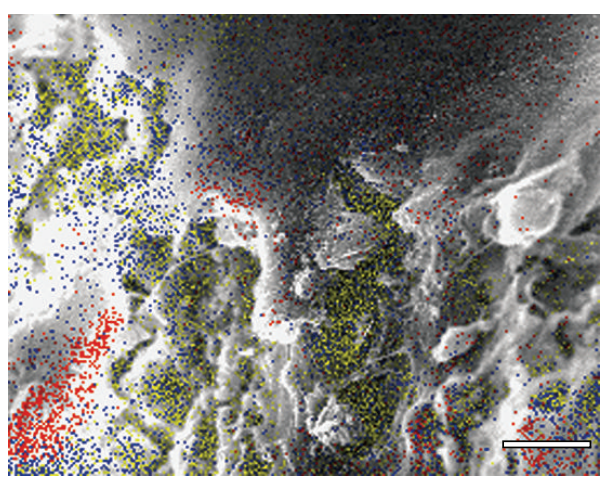

(c)

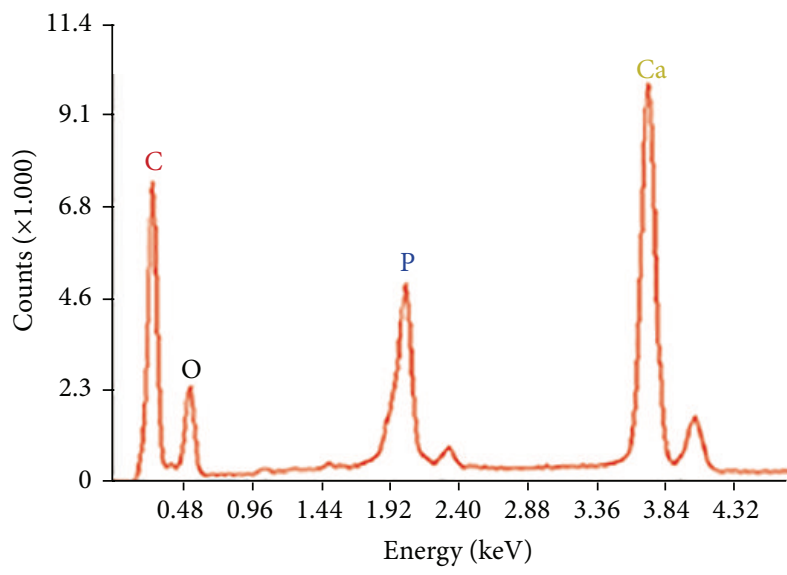

(d)

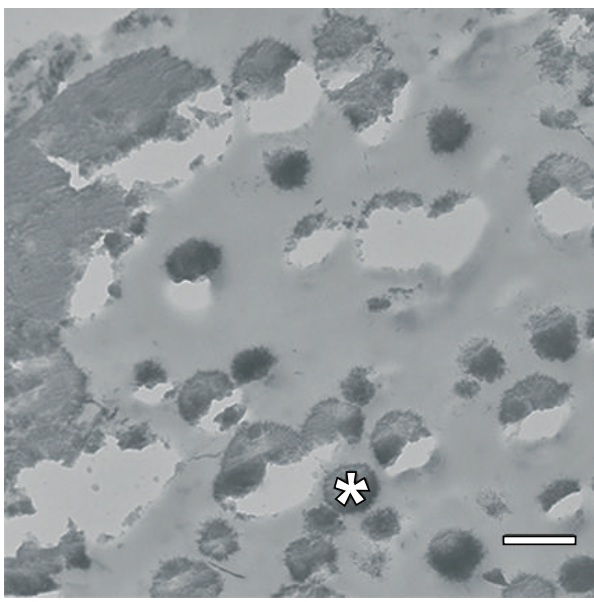

(e)

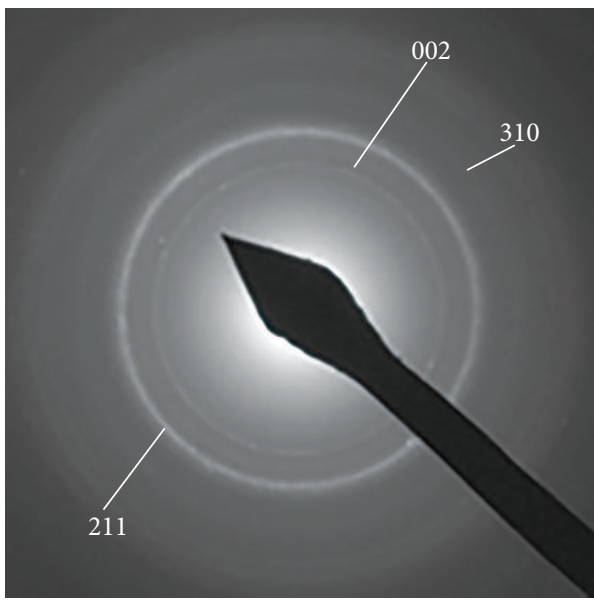

(f)

FIGURE 4: (a) von Kossa staining and counterstaining with methylene blue. Most inner areas of the osteogenically induced GF-iPSC construct showed calcification (asterisks). Some noncalcified fibrous areas were observed (arrow heads). Scale bar: $100 \mu \mathrm{m}$. (b) Magnification of the dotted square in panel (a). The calcified inner area (asterisks) was surrounded by a strongly calcified zone (arrow heads). Arrows indicate osteoid-like tissues. Scale bar: $100 \mu \mathrm{m}$. (c) EDX analyses of the calcified layer area. The yellow, blue, and red dots represent the elemental distribution of calcium, phosphorous, and carbon. Scale bar: $10 \mu \mathrm{m}$. (d) Energy peaks in the EDX graph correspond to elemental phosphorous $(\mathrm{P})$, calcium $(\mathrm{Ca})$, carbon $(\mathrm{C})$, and oxygen $(\mathrm{O})$. (e) TEM image of bone nodules formed in the calcified area (scale bar: $1 \mu \mathrm{m})$. (f) SAED pattern $(002,211$, and 310 rings) of an electron-dense area (asterisk in panel (e)) was indicative of hydroxyapatite. 

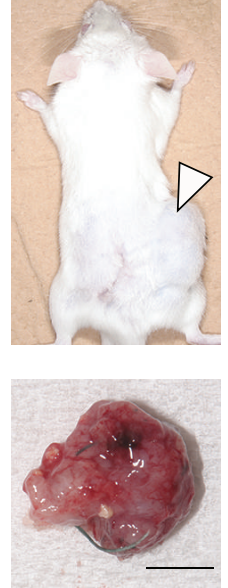

(a)

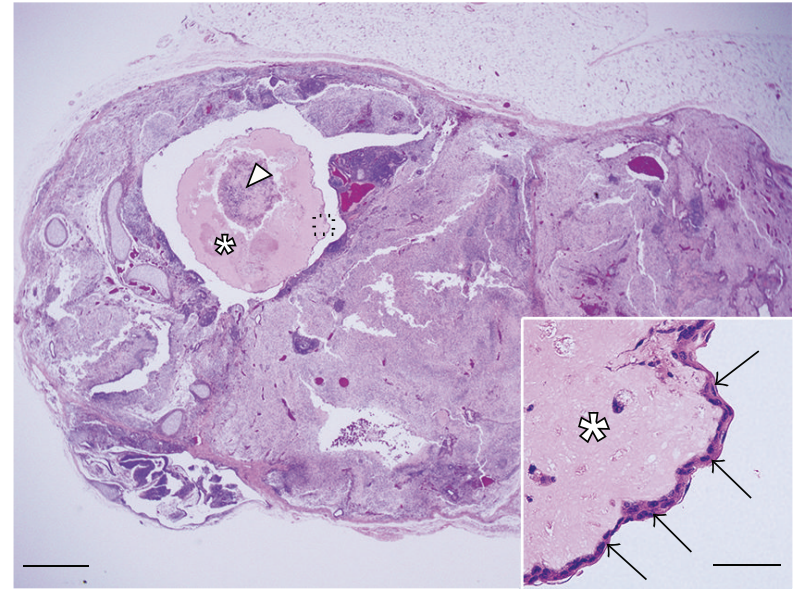

(b)

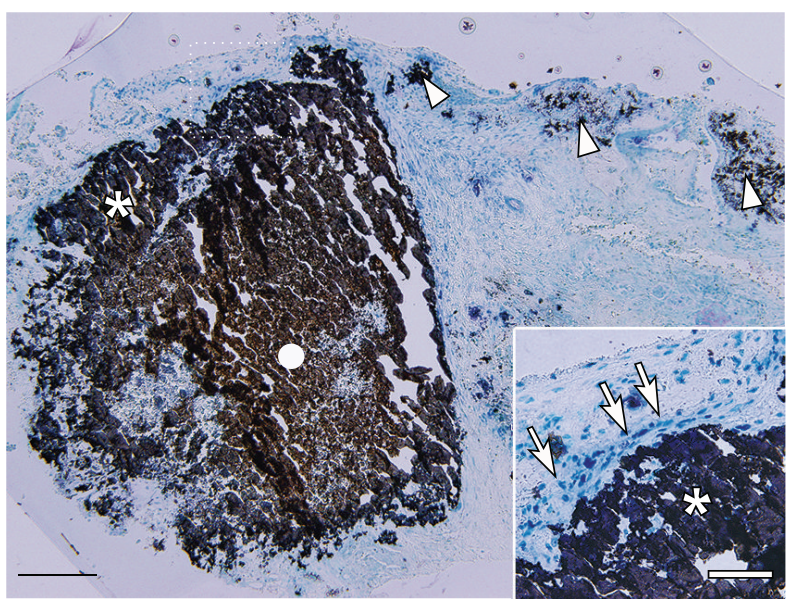

(c)

FIGURE 5: (a) Subcutaneous transplantation of osteogenically induced GF-iPSC constructs into immunodeficient mice resulted in tumor formation (upper panel: arrowhead) at the fourth week. Lower panel: an extracted tumor (scale bar: $1 \mathrm{~cm}$ ). (b) H\&E staining of the extracted transplants indicates teratoma, where the transplanted cell construct (arrowhead) was surrounded by extensive osseous tissue (asterisk). Scale bar: $1 \mathrm{~mm}$. Inset: magnification of the dotted square. Arrows indicate aligned cells on the osseous tissue (asterisk). Scale bar: $50 \mu \mathrm{m}$. (c) von Kossa staining and counterstaining with methylene blue. The transplanted calcified cell construct (circle) and the ectopically formed bone region (asterisk) exhibited robust calcification. Arrowheads indicate sparse calcium deposition in the teratoma. Scale bar: $500 \mu \mathrm{m}$. Inset: magnification of the dotted square. Arrows indicate aligned cells in direct contact with the calcified tissue surface (asterisk) (scale bar: $50 \mu \mathrm{m}$ ).

formation model that is useful for evaluation of bone-forming stem cells and new osteoinductive biomaterials [43]. In this study, we used fibrin gels, which have been shown to be useful for cell delivery [44] and have been used for subcutaneous implantation of MSCs to assess ectopic bone formation [45, 46]. The use of fibrin gels allowed us to deliver the iPSC constructs more easily and in a manner that would retain the constructs at the surgical site during the experimental period. Four weeks after subcutaneous implantation of calcified GF-iPSC constructs, tumor formation was observed at the implanted site (Figure 5(a)). H\&E staining of the extracted transplants indicated teratoma formation (Figure 5(b)), in which tissues of various lineages including extensive cartilage and fibrous osteoid tissue were present. In the teratoma, it should be noted that the transplanted cell constructs were surrounded by a large osseous tissue structure that was covered with aligned cells (Figure 5(b): inset). These aligned cells histologically resembled osteoblasts or bone lining cells, which generate new bone and then remain on its surface [47]. von Kossa staining revealed robust calcification in both the transplanted cell construct and the surrounding osseous tissue region (Figure 5(c)). The aligned cells were in direct contact with the calcified tissue surface (Figure 5(c): inset), implying that they were osteoblast-derived bone lining cells. Sparse calcium deposition was also confirmed in several areas in the teratomas in addition to the cell construct area. These results suggest that the in vitro-synthesized calcified GFiPSC construct contributed to robust ectopic bone formation, although it also elicited teratogenesis.

One critical problem hindering the clinical application of iPSCs is that the contamination of differentiated iPSCs with undifferentiated cells results in teratoma formation 
after transplantation [48]. In this study, methylene blue counterstaining revealed some noncalcified areas in the inner region (Figure 4(a)) where a few undifferentiated or nonosteogenic cells may have remained, thus potentially contributing to teratoma formation. In addition, the fibrin that we used in this study not only is a passive cell delivery matrix but also binds many growth factors, such as fibronectin and von Willebrand factor [49], which have been suggested to be involved in not only bone formation $[50,51]$ but also tumorigenesis [52, 53]. Therefore, the use of fibrin in this study might have indeed partly affected the ectopic bone and teratoma formation of the GF-iPSC constructs. Irradiation of osteogenically induced iPSCs prior to transplantation [54], introduction of a suicide gene into the pluripotency locus [55], or co-treatment with small molecules such as quercetin and YM155 [56] may be useful strategies to prevent tumorigenesis after implantation.

The osteoinductive capacity of the scaffold-free calcified GF-iPSC constructs is attractive for bone tissue engineering, as such an approach would not require the use of additional scaffolds during the transplantation procedure. In particular, the calcified parts of the constructs themselves can be expected to provide a regeneration niche as a scaffolding material, whereas the surrounding aligned osteoblasts would be expected to promote osteoinduction, which may provide a simple and reliable treatment procedure. In addition, the use of temperature-responsive hydrogel molds enables control of shape and size during the fabrication of cell constructs [18]. In a preliminary study, fabrication of larger calcified constructs than the ball-like structure could be achieved using GF-iPSCs; however, formation of such constructs was difficult when mouse MSCs were used in the same system, indicating that iPSCs are advantageous and possibly even that pluripotent cells such as iPSCs are required, for scaffold-free fabrication of large 3D cell constructs.

\section{Conclusions}

This study established size- and shape-controlled mouse GFiPSC constructs by a scaffold-free method using a thermoresponsive hydrogel system. The present data show that mouse GF-iPSCs enable the fabrication of osteoinductive 3D cell constructs, in which the calcified regions and surrounding osteoblasts may function as scaffolds and drivers of osteoinduction, respectively. The fabrication of size- and shape-controlled GF-iPSC constructs, demonstrated to be feasible in the present study, would be advantageous to tailor the calcified GF-iPSC construct to specific bone defects in individual patients. Therefore, scaffold-free calcified GF-iPSC constructs are a promising biological material for iPSC-based bone regenerative therapies, and methods to completely suppress tumorigenesis by the constructs should be explored in future studies.

\section{Competing Interests}

The authors declare that they have no competing interests.

\section{Acknowledgments}

This investigation was supported by a Grant-in-Aid for Scientific Research B25293395 (Hiroshi Egusa) from the Japan Society for the Promotion of Science (JSPS). Support was also received in part from Grants-in-Aid for Scientific Research A25253102 (Hiroshi Egusa, Hiroki Kayashima, and Hirofumi Yatani) from the JSPS.

\section{References}

[1] C. Masaki, T. Nakamoto, T. Mukaibo, Y. Kondo, and R. Hosokawa, "Strategies for alveolar ridge reconstruction and preservation for implant therapy," Journal of Prosthodontic Research, vol. 59, no. 4, pp. 220-228, 2015.

[2] P. Li, Y. Honda, Y. Arima et al., "Interferon- $\gamma$ enhances the efficacy of autogenous bone grafts by inhibiting postoperative bone resorption in rat calvarial defects," Journal of Prosthodontic Research, 2016.

[3] H. Egusa, W. Sonoyama, M. Nishimura, I. Atsuta, and K. Akiyama, "Stem cells in dentistry-part II: clinical applications," Journal of Prosthodontic Research, vol. 56, no. 4, pp. 229-248, 2012.

[4] C. M. Murphy, F. J. O’Brien, D. G. Little, and A. Schindeler, "Cell-scaffold interactions in the bone tissue engineering triad," European Cells and Materials, vol. 26, pp. 120-132, 2013.

[5] S. F. Badylak and T. W. Gilbert, "Immune response to biologic scaffold materials," Seminars in Immunology, vol. 20, no. 2, pp. 109-116, 2008.

[6] H. Egusa, W. Sonoyama, M. Nishimura, I. Atsuta, and K. Akiyama, "Stem cells in dentistry-part I: stem cell sources," Journal of Prosthodontic Research, vol. 56, no. 3, pp. 151-165, 2012.

[7] M. Kaku, Y. Akiba, K. Akiyama, D. Akita, and M. Nishimura, "Cell-based bone regeneration for alveolar ridge augmentation-cell source, endogenous cell recruitment and immunomodulatory function," Journal of Prosthodontic Research, vol. 59, no. 2, pp. 96-112, 2015.

[8] J. H. Lee, J.-H. Seo, K. M. Lee, H.-S. Ryu, and H.-R. Baek, "Fabrication and evaluation of osteoblastic differentiation of human mesenchymal stem cells on novel cao- $\mathrm{SiO}_{2}-\mathrm{P}_{2} \mathrm{O}_{5}-\mathrm{B}_{2} \mathrm{O}_{3}$ glass-ceramics," Artificial Organs, vol. 37, no. 7, pp. 637-647, 2013.

[9] J. Zhou, C. Xu, G. Wu et al., "In vitro generation of osteochondral differentiation of human marrow mesenchymal stem cells in novel collagen-hydroxyapatite layered scaffolds," Acta Biomaterialia, vol. 7, no. 11, pp. 3999-4006, 2011.

[10] J.-I. Sasaki, T. Matsumoto, H. Egusa et al., "In vitro reproduction of endochondral ossification using a 3D mesenchymal stem cell construct," Integrative Biology, vol. 4, no. 10, pp. 1207-1214, 2012.

[11] M. Kittaka, M. Kajiya, H. Shiba et al., "Clumps of a mesenchymal stromal cell/extracellular matrix complex can be a novel tissue engineering therapy for bone regeneration," Cytotherapy, vol. 17, no. 7, pp. 860-873, 2015.

[12] Z.-Y. Zhang, S.-H. Teoh, M. S. K. Chong et al., "Superior osteogenic capacity for bone tissue engineering of fetal compared with perinatal and adult mesenchymal stem cells," STEM CELLS, vol. 27, no. 1, pp. 126-137, 2009.

[13] K. Takahashi and S. Yamanaka, "Induction of pluripotent stem cells from mouse embryonic and adult fibroblast cultures by defined factors," Cell, vol. 126, no. 4, pp. 663-676, 2006. 
[14] H. Egusa, K. Okita, H. Kayashima et al., "Gingival fibroblasts as a promising source of induced pluripotent stem cells," PloS ONE, vol. 5, no. 9, Article ID e12743, 2010.

[15] G. Yu, H. Okawa, K. Okita et al., "Gingival fibroblasts as autologous feeders for induced pluripotent stem cells," Journal of Dental Research, vol. 95, no. 1, pp. 110-118, 2016.

[16] X. Lou, "Induced pluripotent stem cells as a new strategy for osteogenesis and bone regeneration," Stem Cell Reviews and Reports, vol. 11, no. 4, pp. 645-651, 2015.

[17] H. Egusa, H. Kayashima, J. Miura et al., "Comparative analysis of mouse-induced pluripotent stem cells and mesenchymal stem cells during osteogenic differentiation in vitro," Stem Cells and Development, vol. 23, no. 18, pp. 2156-2169, 2014.

[18] J.-I. Sasaki, T.-A. Asoh, T. Matsumoto et al., "Fabrication of three-dimensional cell constructs using temperatureresponsive hydrogel," Tissue Engineering Part A, vol. 16, no. 8, pp. 2497-2504, 2010.

[19] M. Y. Speer, X. Li, P. G. Hiremath, and C. M. Giachelli, "Runx2/Cbfal, but not loss of myocardin, is required for smooth muscle cell lineage reprogramming toward osteochondrogenesis," Journal of Cellular Biochemistry, vol. 110, no. 4, pp. 935-947, 2010.

[20] J. L. Fowlkes, R. C. Bunn, L. Liu et al., "Runt-related transcription factor 2 (RUNX2) and RUNX2-related osteogenic genes are down-regulated throughout osteogenesis in type 1 diabetes mellitus," Endocrinology, vol. 149, no. 4, pp. 1697-1704, 2008.

[21] L. A. Kaback, D. Y. Soung, A. Naik et al., "Teriparatide (134 human $\mathrm{PTH}$ ) regulation of osterix during fracture repair," Journal of Cellular Biochemistry, vol. 105, no. 1, pp. 219-226, 2008.

[22] J. Jadlowiec, H. Koch, X. Zhang, P. G. Campbell, M. Seyedain, and C. Sfeir, "Phosphophoryn regulates the gene expression and differentiation of NIH3T3, MC3T3-E1, and human mesenchymal stem cells via the integrin/MAPK signaling pathway," The Journal of Biological Chemistry, vol. 279, no. 51, pp. 53323-53330, 2004.

[23] G. Gautier, M. Humbert, F. Deauvieau et al., "A type I interferon autocrine-paracrine loop is involved in Toll-like receptorinduced interleukin-12p70 secretion by dendritic cells," The Journal of Experimental Medicine, vol. 201, no. 9, pp. 1435-1446, 2005.

[24] J. Sasaki, M. Hashimoto, S. Yamaguchi et al., "Fabrication of biomimetic bone tissue using mesenchymal stem cell-derived three-dimensional constructs incorporating endothelial cells," PLoS ONE, vol. 10, no. 6, Article ID e0129266, 2015.

[25] T. D. Schmittgen and K. J. Livak, "Analyzing real-time PCR data by the comparative $C_{\mathrm{T}}$ method," Nature Protocols, vol. 3, no. 6, pp. 1101-1108, 2008.

[26] H. Egusa, M. Kobayashi, T. Matsumoto, J.-I. Sasaki, S. Uraguchi, and H. Yatani, "Application of cyclic strain for accelerated skeletal myogenic differentiation of mouse bone marrowderived mesenchymal stromal cells with cell alignment," Tissue Engineering-Part A, vol. 19, no. 5-6, pp. 770-782, 2013.

[27] K. Tashiro, M. Inamura, K. Kawabata et al., "Efficient adipocyte and osteoblast differentiation from mouse induced pluripotent stem cells by adenoviral transduction," Stem Cells, vol. 27 , no. 8 , pp. 1802-1811, 2009.

[28] G. Bilousova, D. H. Jun, K. B. King et al., "Osteoblasts derived from induced pluripotent stem cells form calcified structures in scaffolds both in vitro and in vivo," Stem Cells, vol. 29, no. 2, pp. 206-216, 2011.
[29] K. Nakashima, X. Zhou, G. Kunkel et al., "The novel zinc fingercontaining transcription factor Osterix is required for osteoblast differentiation and bone formation," Cell, vol. 108, no. 1, pp. 1729, 2002.

[30] T. M. Liu and E. H. Lee, "Transcriptional regulatory cascades in Runx2-dependent bone development," Tissue EngineeringPart B: Reviews, vol. 19, no. 3, pp. 254-263, 2013.

[31] M. Kaku and M. Yamauchi, "Mechano-regulation of collagen biosynthesis in periodontal ligament," Journal of Prosthodontic Research, vol. 58, no. 4, pp. 193-207, 2014.

[32] A. Patti, L. Gennari, D. Merlotti, F. Dotta, and R. Nuti, "Endocrine actions of osteocalcin," International Journal of Endocrinology, vol. 2013, Article ID 846480, 10 pages, 2013.

[33] T. Matsubara, K. Kida, A. Yamaguchi et al., "BMP2 regulates osterix through Msx2 and Runx2 during osteoblast differentiation," The Journal of Biological Chemistry, vol. 283, no. 43, pp. 29119-29125, 2008.

[34] D.-S. Lee, H.-W. Choung, H.-J. Kim et al., "NFI-C regulates osteoblast differentiation via control of osterix expression," Stem Cells, vol. 32, no. 9, pp. 2467-2479, 2014.

[35] M.-H. Lee, T.-G. Kwon, H.-S. Park, J. M. Wozney, and H.M. Ryoo, "BMP-2-induced Osterix expression is mediated by Dlx 5 but is independent of Runx2," Biochemical and Biophysical Research Communications, vol. 309, no. 3, pp. 689-694, 2003.

[36] L. Szabova, S. S. Yamada, H. Wimer et al., "MT1-MMP and type II collagen specify skeletal stem cells and their bone and cartilage progeny," Journal of Bone and Mineral Research, vol. 24, no. 11, pp. 1905-1916, 2009.

[37] E. Bonucci, "Bone mineralization," Frontiers in Bioscience, vol. 17, no. 1, pp. 100-128, 2012.

[38] M. Ejtehadifar, K. Shamsasenjan, A. Movassaghpour et al., "The effect of hypoxia on mesenchymal stem cell biology," Advanced Pharmaceutical Bulletin, vol. 5, no. 2, pp. 141-149, 2015.

[39] M. Wagegg, T. Gaber, F. L. Lohanatha et al., "Hypoxia promotes osteogenesis but suppresses adipogenesis of human mesenchymal stromal cells in a hypoxia-inducible factor-1 dependent manner," PLoS ONE, vol. 7, no. 9, Article ID e46483, 2012.

[40] A. Idelevich, Y. Rais, and E. Monsonego-Ornan, "Bone Gla protein increases HIF- $1 \alpha$-dependent glucose metabolism and induces cartilage and vascular calcification," Arteriosclerosis, Thrombosis, and Vascular Biology, vol. 31, no. 9, pp. e55-e71, 2011.

[41] Y. K. Kim, L.-S. Gu, T. E. Bryan et al., "Mineralisation of reconstituted collagen using polyvinylphosphonic acid/polyacrylic acid templating matrix protein analogues in the presence of calcium, phosphate and hydroxyl ions," Biomaterials, vol. 31, no. 25, pp. 6618-6627, 2010.

[42] F. Langenbach, C. Naujoks, P. V. Kersten-Thiele et al., "Osteogenic differentiation influences stem cell migration out of scaffold-free microspheres," Tissue Engineering Part A, vol. 16, no. 2, pp. 759-766, 2010.

[43] M. A. Scott, B. Levi, A. Askarinam et al., "Brief review of models of ectopic bone formation," Stem Cells and Development, vol. 21, no. 5, pp. 655-667, 2012.

[44] P. A. Janmey, J. P. Winer, and J. W. Weisel, "Fibrin gels and their clinical and bioengineering applications," Journal of the Royal Society Interface, vol. 6, no. 30, pp. 1-10, 2009.

[45] C. Jaquiéry, S. Schaeren, J. Farhadi et al., "In vitro osteogenic differentiation and in vivo bone-forming capacity of human isogenic jaw periosteal cells and bone marrow stromal cells," Annals of Surgery, vol. 242, no. 6, pp. 859-868, 2005. 
[46] J. Sasaki, T. Matsumoto, and S. Imazato, "Oriented bone formation using biomimetic fibrin hydrogels with three-dimensional patterned bone matrices," Journal of Biomedical Materials Research Part A, vol. 103, no. 2, pp. 622-627, 2015.

[47] F. Long, "Building strong bones: molecular regulation of the osteoblast lineage," Nature Reviews Molecular Cell Biology, vol. 13, no. 1, pp. 27-38, 2012.

[48] B. Zhong, K. L. Watts, J. L. Gori et al., "Safeguarding nonhuman primate iPS cells with suicide genes," Molecular Therapy, vol. 19, no. 9, pp. 1667-1675, 2011.

[49] J. W. Weisel, "Fibrinogen and fibrin," Advances in Protein Chemistry, vol. 70, pp. 247-299, 2005.

[50] D. Kaigler, Z. Wang, K. Horger, D. J. Mooney, and P. H. Krebsbach, "VEGF scaffolds enhance angiogenesis and bone regeneration in irradiated osseous defects," Journal of Bone and Mineral Research, vol. 21, no. 5, pp. 735-744, 2006.

[51] A. Bentmann, N. Kawelke, D. Moss et al., "Circulating fibronectin affects bone matrix, whereas osteoblast fibronectin modulates osteoblast function," Journal of Bone and Mineral Research, vol. 25, no. 4, pp. 706-715, 2010.

[52] V. Terraube, I. Marx, and C. V. Denis, "Role of von Willebrand factor in tumor metastasis," Thrombosis Research, vol. 120, no. 2, pp. S64-S70, 2007.

[53] A. von Au, M. Vasel, S. Kraft et al., "Circulating fibronectin controls tumor growth," Neoplasia, vol. 15, no. 8, pp. 925-938, 2013.

[54] T. Hayashi, H. Misawa, H. Nakahara et al., "Transplantation of osteogenically differentiated mouse iPS cells for bone repair," Cell Transplantation, vol. 21, no. 2-3, pp. 591-600, 2012.

[55] F. Cheng, Q. Ke, F. Chen et al., "Protecting against wayward human induced pluripotent stem cells with a suicide gene," Biomaterials, vol. 33, no. 11, pp. 3195-3204, 2012.

[56] M.-O. Lee, S. H. Moon, H.-C. Jeong et al., "Inhibition of pluripotent stem cell-derived teratoma formation by small molecules," Proceedings of the National Academy of Sciences of the United States of America, vol. 110, no. 35, pp. E3281-E3290, 2013. 

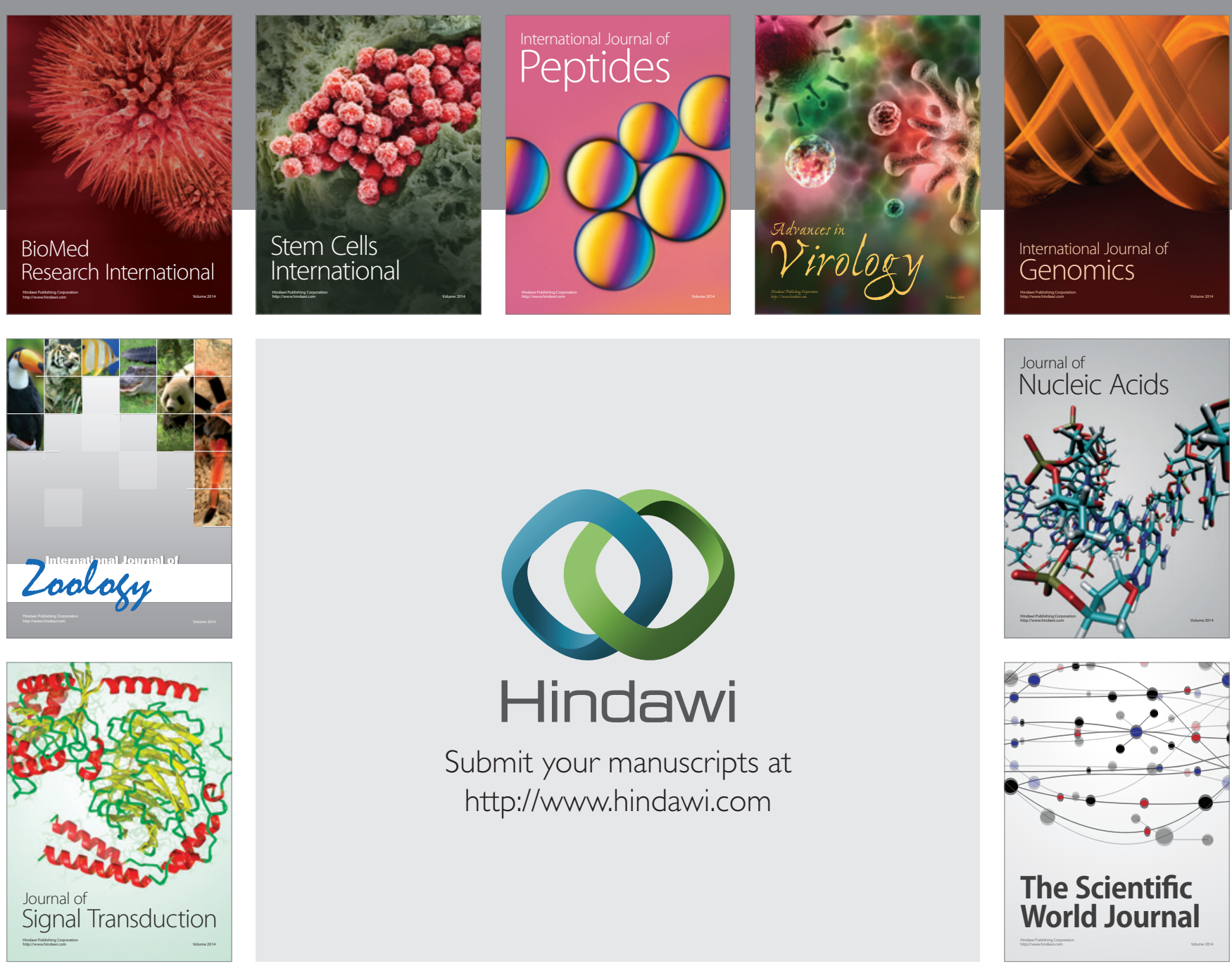

Submit your manuscripts at

http://www.hindawi.com
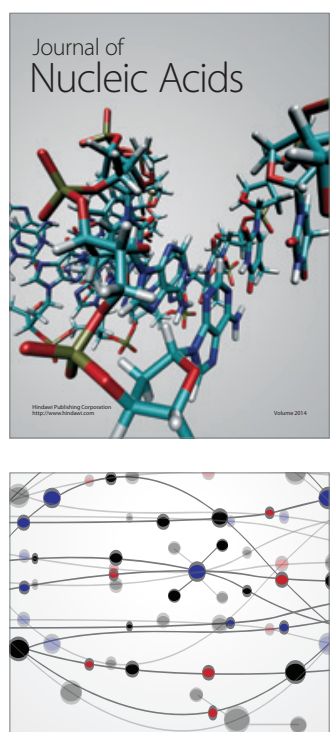

The Scientific World Journal
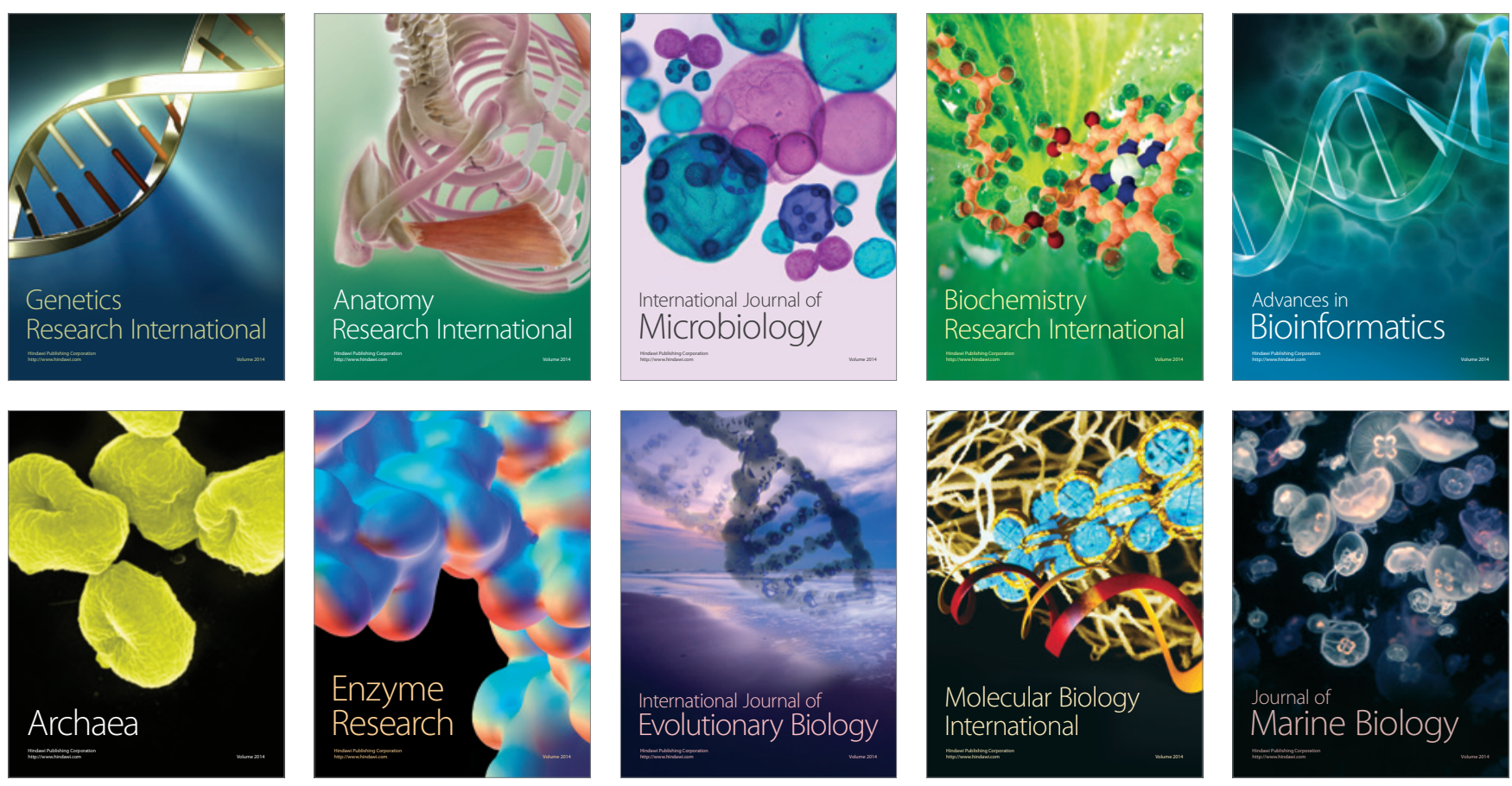